\title{
Renalase - a potential biomarker for risk of atrial fibrillation?
}

\author{
Markus P. Schlaich ${ }^{1,2}$, Gavin W. Lambert ${ }^{2,3}$, Nina Eikelis ${ }^{2,3}$ \\ 'Dobney Hypertension Centre, School of Medicine and Pharmacology, Royal Perth Hospital Unit, University of Western Australia, Perth, Australia \\ ${ }^{2}$ Neurovascular Hypertension and Kidney Disease and Human Neurotransmitter Laboratories, Baker Heart and Diabetes Institute, Melbourne, \\ Australia \\ ${ }^{3}$ Iverson Health Innovation Research Institute, Faculty of Health, Arts and Design, Swinburne University of Technology, Melbourne, Australia
}

Article Wybraniec et al., see p. 1232

Pulmonary vein isolation (PVI) is now one of the standard procedures used to treat atrial fibrillation (AF) [1]. However, a single ablation procedure has been shown to be effective in only about $50 \%$ of patients at three-year follow-up and multiple procedures were required to achieve control of $\mathrm{AF}$ in $\sim 80 \%$ of sufferers [2]. Furthermore, the success rates differ between patients presenting with paroxysmal $(60 \%-80 \%)$ and persistent $\mathrm{AF}(50 \%-70 \%)$. As with any catheter-based procedure there are inherent risks pertaining to access site and vascular complications, pericarditis, and others. A recent report from a large registry revealed a complication rate of $10.5 \%$ defined as any procedure-related adverse event resulting in permanent injury or death, requiring intervention or treatment, or prolonging/requiring hospitalisation for $>48 \mathrm{~h}$ [3]. These results highlight the need for better predictors of procedural success in order to minimise exposure of patients who are unlikely to benefit from the procedure. Therefore, cardiologists have long sought various anatomical or biological factors that can determine which patients may be preferred candidates for PVI. Widely and easily accessible biomarkers would best serve this purpose. A recent report summarises the currently available evidence with focus on myocardial injury biomarkers, natriuretic peptides, inflammatory markers, oxidative stress biomarkers and microRNAs [4].

In this context, Wybraniec et al. [5] in the current issue of the journal report on the association between the levels of a catecholamine-metabolising enzyme, renalase, and clinical characteristics of AF. Their cohort comprised 69 patients undergoing PVI for either paroxysmal $(n=62)$ or persistent $(n=7) \mathrm{AF}$ and 15 matched controls. The participants underwent electrocardiographic Holter monitoring to confirm (study group) or exclude (control group) AF. The study group underwent a second seven-day electrocardiogram monitoring six months after the ablation procedure to assess the response to $\mathrm{PVI}$ and to detect possible recurrent AF. Further tests performed prior to the PVI procedure included transthoracic echocardiography with speckle tracking imaging to assess left atrial (LA) global longitudinal strain. To determine renalase plasma concentrations the authors used a commercially available ELISA.

Wybraniec et al. found that low plasma renalase concentration was inversely related to impaired rate control, higher AF burden and advanced LA remodelling. However, renalase concentration did not predict the maintenance of sinus rhythm at six months after the PVI procedure [5]. Accordingly, the authors appropriately conclude that plasma renalase concentration failed to predict recurrence of $\mathrm{AF}$ in the six-month observation.

While the initial hypothesis of the potential utility of renalase to predict AF recurrence may be disproven, the study provides some interesting insights. Renalase was first described by Desir et al. [6] as an extracellular amine oxidase. While it had been thought to be expressed primarily in the kidney, synthesis of renalase was also demonstrated in the brain and peripheral nerves [7]. Such an expression pattern would be in line with its postulated mode of action to degrade circulating catecholamines and links it to the regulation of sympathetic nervous system activity [8]. Although there has been some doubt in the literature on the "true" function of renalase [9], a number of studies showed its negative association with blood pressure [10, 11].

Interestingly, the authors noted that while low levels of renalase were associated with increased burden of $\mathrm{AF}$, the 
study group as a whole had significantly higher renalase levels compared to age- and sex-matched control subjects with similar comorbidities [5]. These findings are difficult to interpret since no other studies have measured plasma concentrations of renalase in patient cohorts with AF. Questions that need to be addressed relate to the reliability of the assay used, the absolute and normal values in various patient cohorts, as well as the relation of these renalase concentrations to other associated markers, in particular catecholamines. Unfortunately, catecholamine levels were not measured in the current study, which would have helped to determine whether the proposed association between (relatively) reduced renalase levels and higher concentrations of various catecholamines could be confirmed. Furthermore, assessment of any relation to the degree of kidney function would have been relevant, since impaired kidney function has been associated with reduced renalase levels, thereby potentially driving the well-established sympathetic overdrive in chronic kidney disease [8]. Of note, sympathetic activation has clearly been associated with cardiac arrhythmias, and therapeutic approaches specifically targeting sympathetic overactivity, such as renal denervation, have been shown to beneficially impact LA remodelling [12].

Increased renalase concentration in a given study cohort compared to a matched control group may be a reflection of disease progression. It is possible that initially renalase levels increase to counteract the cardiovascular consequences and the cardiac remodelling associated with AF. However, as the disease sets in, renalase can become exhausted and therefore circulating levels may decrease over time. The observation that renalase levels were lower in patients with persistent AF compared to paroxysmal AF $(19.05 \pm 12.60$ vs. $28.77 \pm 9.48 \mu \mathrm{g} / \mathrm{mL})$ may be considered as supporting evidence, however, the number of patients with persistent AF was very small $(n=7)$. Future studies should therefore compare renalase levels between newly diagnosed AF patients and those with longer history of AF and should also compare paroxysmal and persistent AF. Longitudinal assessment of renalase concentrations in concert with relevant biomarkers presumably affected by altered renalase availability such as catecholamines may provide further insight into its role in patients with AF. Furthermore, given its enzymatic nature, assessment of renalase activity rather than its absolute abundance in plasma may help to shed some light on its potential role in the context of AF.

As it stands, assessment of renalase plasma concentrations does not appear to be useful to predict recurrence of AF within a six-month observation period. However, it may have utility as a biomarker of the risk of AF and potentially as a marker of progression of the disease. Research into this important area should therefore continue and address the questions raised above.
Conflict of interest: Dr. Markus P. Schlaich is an investigator in studies sponsored by Medtronic and his laboratories have received research funding from Medtronic. He serves on scientific advisory boards for Abbott (formerly Solvay) Pharmaceuticals, Boehringer Ingelheim, Novartis Pharmaceuticals, and Medtronic and has received honoraria and travel support from Abbott, Boehringer Ingelheim, Servier, Novartis, and Medtronic. Dr. Gavin W. Lambert has acted as a consultant for Medtronic and has received honoraria or travel support for presentations from Pfizer, Wyeth Pharmaceuticals, Servier and Medtronic. Dr. Nina Eikelis has nothing to disclose.

\section{References}

1. Pappone C, Rosanio S, Oreto G, et al. Circumferential radiofrequency ablation of pulmonary vein ostia: A new anatomic approach for curing atrial fibrillation. Circulation. 2000; 102(21): 2619-2628, indexed in Pubmed: 11085966.

2. Ganesan AN, Shipp NJ, Brooks AG, et al. Long-term outcomes of catheter ablation of atrial fibrillation: a systematic review and meta-analysis. J Am Heart Assoc. 2013; 2(2): e004549, doi: 10.1161/JAHA.112.004549, indexed in Pubmed: 23537812.

3. De Greef Y, Ströker E, Schwagten B, et al. Complications of pulmonary vein isolation in atrial fibrillation: predictors and comparison between four different ablation techniques: Results from the MIddelheim PVI-registry. Europace. 2017 [Epub ahead of print], doi: 10.1093/europace/eux233, indexed in Pubmed: 29016870.

4. Tsiachris D, Giannopoulos G, Kossyvakis C, et al. Biomarkers determining prognosis of atrial fibrillation ablation. Curr Med Chem. 2018 [Epub ahead of print], doi: 10.2174/092986732566 6180320122930, indexed in Pubmed: 29557741.

5. Wybraniec MT, Wieczorek J, Woźniak-Skowerska I, et al. Renalase is associated with adverse left atrial remodelling and disease burden in patients with atrial fibrillation undergoing pulmonary vein isolation. Kardiol Pol. 2018; 76(8): 1232-1241, doi: 10.5603/KP.a2018.0108, indexed in Pubmed: 29944173.

6. $\mathrm{Xu} \mathrm{J,} \mathrm{Li} \mathrm{G,} \mathrm{Wang} \mathrm{P,} \mathrm{et} \mathrm{al.} \mathrm{Renalase} \mathrm{is} \mathrm{a} \mathrm{novel,} \mathrm{soluble} \mathrm{monoamine}$ oxidase that regulates cardiac function and blood pressure. J Clin Invest. 2005; 115(5): 1275-1280, doi: 10.1172/JCI24066, indexed in Pubmed: 15841207.

7. Hennebry SC, Eikelis N, Socratous F, et al. Renalase, a novel soluble FAD-dependent protein, is synthesized in the brain and peripheral nerves. Mol Psychiatry. 2010; 15(3): 234-236, doi: 10.1038/mp.2009.74, indexed in Pubmed: 20168325.

8. Schlaich MP, Socratous F, Hennebry S, et al. Sympathetic activation in chronic renal failure. J Am Soc Nephrol. 2009; 20(5): 933-939, doi: 10.1681/ASN.2008040402, indexed in Pubmed: 18799718.

9. Eikelis N, Hennebry S, Lambert G, et al. Does renalase degrade catecholamines? Kidney International. 2011; 79(12): 1380-1381, doi: 10.1038/ki.2011.90, indexed in Pubmed: 21625261.

10. Desir GV. Role of renalase in the regulation of blood pressure and the renal dopamine system. Curr Opin Nephrol Hypertens. 2011; 20(1): 31-36, doi: 10.1097/MNH.0b013e3283412721, indexed in Pubmed: 21099685.

11. Lv YB, Wang Y, Ma WG, et al. Association of Renalase SNPs rs2296545 and rs2576178 with the Risk of Hypertension: A Meta-Analysis. PLoS One. 2016; 11(7): e0158880, doi: 10.1371/journal.pone.0158880, indexed in Pubmed: 27434211.

12. McLellan AJA, Schlaich MP, Taylor AJ, et al. Reverse cardiac remodeling after renal denervation: Atrial electrophysiologic and structural changes associated with blood pressure lowering. Heart Rhythm. 2015; 12(5): 982-990, doi: 10.1016/j.hrthm.2015.01.039, indexed in Pubmed: 25638699. 